\title{
Estudo etnobotânico em quintais agroflorestais em bairro na Cidade de Cuiabá, Mato Grosso
}

\section{Ethnobotanical study in agroforestry homegardens in a neighborhood in the City of Cuiabá, Mato Grosso}

\author{
Carla Spiller ${ }^{1}$, Maria de Fatima Barbosa Coelho ${ }^{* 2}$, Vanessa Damasceno Gonçalves ${ }^{3}$, Ludmila Porto Piton ${ }^{4}$; Elisangela \\ Clarete Camili ${ }^{5}$
}

Resumo: Os quintais são espaços de uso, manejo e conservação de diferentes espécies vegetais contribuindo para a renda familiar e segurança alimentar das famílias. O objetivo no presente estudo foi fazer um levantamento das espécies em quintais do Bairro Jardim Florianópolis em Cuiabá, Mato Grosso. A pesquisa foi conduzida através de entrevistas semiestruturadas, associadas às técnicas de "bola de neve" e turnê-guiada. Foram registradas 58 espécies pertencentes a 52 gêneros e 27 famílias, destacando-se as famílias Arecaceae (7 spp.), Araceae (5 spp.), Liliaceae (5 spp.) Myrtaceae (4 spp.) e Rubiaceae (4 spp.). A maior parte das espécies são exóticas $(75,86 \%)$. As plantas são classificadas pelos moradores em ornamentais, alimentícias e medicinais. O uso ornamental representou $62,5 \%$, o medicinal 21,5\% e o alimentício apenas $16 \%$. As espécies ornamentais de hábito herbáceo são as mais importantes com predominância da família Arecaceae.

Palavras-chave: Agricultura urbana; Arecaceae; Cerrado

\begin{abstract}
Homegardens are spaces for the use, management and conservation of different plant species contributing to family income and household food security. The aim of this study was to survey the species in homegardens of Jardim Florianópolis neighborhood in Cuiabá, Mato Grosso. The research was conducted through semi-structured interviews, associated with snowball and tour-guided techniques. A total of 58 species belonging to 52 genera and 27 families were recorded, including the families Arecaceae (7 spp.), Araceae (5 spp.), Liliaceae (5 spp.) Myrtaceae (4 spp.) and Rubiaceae. Most species are exotic (75.86\%). The ornamental use represented $62.5 \%$, the medicinal $21.5 \%$ and the food only $16 \%$. The ornamental species of herbaceous habit are the most important with predominance of the family Arecaceae.
\end{abstract}

Key words: Urban agriculture; Arecaceae; Cerrado

\footnotetext{
*Autor para correspondência

Recebido para publicação em 27/11/2015; aprovado em 20/10/2016

${ }_{1,3,4}$ Mestre, Programa de Pós-Graduação em Agricultura Tropical (PPGAT), Av. Fernando Correa da Costa, nº 2367 - Cidade Universitária - $78060-900$ Cuiabá - MT, Brasil, Telefone/Fax +55(65) 36158618 E-mail: vanessa-d-goncalves@ hotmail.com, ludy_porto@ hotmail.com

${ }^{2,5}$ Profa. Titular, Doutora, Universidade Federal de Mato Grosso (UFMT), Faculdade de Agronomia, Medicina Veterinária e Zootecnia (FAMEVZ), Programa de Pós-Graduação em Agricultura Tropical (PPGAT), Av. Fernando Correa da Costa, nº 2367 - Cidade Universitária - 78060-900 - Cuiabá - MT, Brasil , Telefone/Fax +55(65) 36158618,E-mail:coelhomfstrela@gmail.com, eccamili@hotmail.com
} 


\section{INTRODUÇÃO}

Os sistemas agroflorestais denominados quintais no Brasil são formas de uso da terra em que varias espécies de árvores são cultivadas, juntamente com culturas anuais e a criação de pequenos animais ao redor da casa. Estes espaços são mantidos pela família e os produtos são para atender as necessidades nutricionais da família, bem como a de energia e saúde (KUMAR; NAIR, 2006).

A importância dos quintais agroflorestais está relacionada com eficiência do uso da terra, conservação de espécies e sustentabilidade (KUMAR; NAIR, 2006; FLORENTINO et al., 2007), com a segurança alimentar e aumento da renda familiar, introdução e domesticação de espécies (GAO et al., 2012). Os quintais são importantes na conservação de plantas medicinais por comunidades rurais e urbanas (BAJPAI et al., 2013; PERNA; LAMANOFERREIRA, 2014).

Os quintais são uma das formas mais antigas de manejo da terra, fato esse que, por si só, indica sua sustentabilidade. Embora esse sistema de produção de múltiplas espécies tenha provido e sustentado milhões de pessoas economicamente, pouca atenção científica tem sido destinada ao assunto (AMARAL NETO, 2008).

No Estado de Mato Grosso alguns estudos já foram realizados abordando o tema quintais (AMARAL; GUARIM NETO, 2008; GUARIM NETO; CARNIELLO, 2008; CARNIELLO et al., 2010; PASA, 2011; MOREIRA; GUARIM NETO, 2015). Infelizmente esses quintais estão desaparecendo do cenário urbano, à medida que a especulação imobiliária vem avançando com seus edifícios de concreto, com um mínimo de área verde disponível para amenizar o calor típico da região, além do espaço de convivência com a família e amigos e lazer das crianças.

No presente estudo objetivou-se realizar um levantamento das espécies vegetais usadas em quintais agroflorestais urbanos no Bairro Jardim Florianópolis em Cuiabá, Mato Grosso.

\section{MATERIAL E MÉTODOS}

O estudo foi desenvolvido no Bairro Jardim Florianópolis situado na porção norte de Cuiabá, Mato Grosso, nas proximidades da rodovia Emanuel Pinheiro e sofreu expansão a partir da década de 80 por conta da construção do Centro Político Administrativo (CARMO, 2014).

Foram selecionadas com a técnica bola de neve (BAILEY, 1994) quinze pessoas responsáveis por seus quintais e realizadas entrevistas semiestruturadas. Depois foram visitas guiadas nos quinze quintais (ALBUQUERQUE et al., 2014) com os principais envolvidos no manejo e manutenção dos quintais para confirmar as espécies citadas e coletar amostras. O tempo médio de cada entrevista foi de 60 minutos. Em cada quintal foram feitos levantamentos das espécies vegetais com a coleta de amostras para exsicatas e identificação no Herbário da Universidade Federal de Mato Grosso.

As espécies foram classificadas quanto ao uso de acordo com as definições dos próprios entrevistados, evitando-se utilizar categorias pré-definidas. À medida que foram coletadas, as informações foram compiladas sob a forma de um banco de dados, utilizando-se matrizes de textos, conforme metodologia proposta por (AMOROZO; VIERTLER, 2010).

\section{RESULTADOS E DISCUSSÃO}

Nos quintais foram encontradas 27 famílias e 56 espécies distribuídas em 54 gêneros (Tabela 1). A maioria das espécies são exóticas $(75,86 \%)$ e isto se deve ao seu uso como ornamental, pois os entrevistados relataram que foram obtidas em viveiros todas as espécies ornamentais, e estes viveiros não trabalham com plantas nativas.

Tabela 1. Características das espécies levantadas nos quintais do Bairro Jardim Florianópolis em Cuiabá, Mato Grosso

\begin{tabular}{|c|c|c|c|c|}
\hline Família/espécie & Nome vernacular & Origem & Uso & $\begin{array}{l}\text { Habito de } \\
\text { crescimento }\end{array}$ \\
\hline \multicolumn{5}{|l|}{ AMARYLLIDACEAE } \\
\hline Agave augustifolia Haw. & agave de dragão & $\mathrm{E}$ & ornamental & erva \\
\hline Agave attenuata Salm-Dyck & piteira & $\mathrm{E}$ & ornamental & erva \\
\hline \multicolumn{5}{|l|}{ ANACARDIACEAE } \\
\hline Anacardium ocidentalis $\mathrm{L}$. & cajueiro & $\mathrm{N}$ & alimentícia & árvore \\
\hline Mangifera indica $\mathrm{L}$. & mangueira & $\mathrm{E}$ & alimentícia & árvore \\
\hline \multicolumn{5}{|l|}{ ANNONACEAE } \\
\hline Annona squamosa $\mathrm{L}$. & ata & $\mathrm{N}$ & alimentícia & árvore \\
\hline \multicolumn{5}{|l|}{ APOCYNACEAE } \\
\hline Allamandra neriifolia A. Schottii & alamanda & $\mathrm{E}$ & ornamental & erva \\
\hline \multicolumn{5}{|l|}{ ARACEAE } \\
\hline Anthurium andraeanum Linden & antúrio & $\mathrm{E}$ & ornamental & erva \\
\hline Dieffenbachia seguine (Jacq.) Schot. & comigo ninguém pode & $\mathrm{E}$ & ornamental & erva \\
\hline Monstera deliciosa $\mathrm{L}$. & costela de adão & E & ornamental & erva \\
\hline Scindapsus pictus Argyraeus & jiboia & $\mathrm{E}$ & ornamental & erva \\
\hline Spathiphyllum wallisii Regel. & lirio & E & ornamental & erva \\
\hline \multicolumn{5}{|l|}{ ARECACEAE } \\
\hline Chrysalidocarpus lutescens $\mathrm{H}$. Wendl. & areca bambu & E & ornamental & árvore \\
\hline Roystonea oleraceae (Jacq.) O.F. Cook & palmeira imperial & $\mathrm{E}$ & ornamental & árvore \\
\hline Trithrinax brasiliensis Mart & palmeira leque & $\mathrm{E}$ & ornamental & árvore \\
\hline Cocos nucifera $\mathrm{L}$. & coqueiro & $\mathrm{E}$ & alimenticia & árvore \\
\hline Phoenix roebelenii $\mathrm{O}^{\prime} \mathrm{Brien}$ & fênix & $\mathrm{E}$ & ornamental & árvore \\
\hline Licuala grandis $\mathrm{L}$. & licuala & $\mathrm{E}$ & ornamental & árvore \\
\hline Yucca brevifolia $\mathrm{L}$. & yuca & $\mathrm{E}$ & ornamental & árvore \\
\hline \multicolumn{5}{|l|}{ CACTACEAE } \\
\hline Opuntia ficus-indica Mill. & cacto & $\mathrm{N}$ & ornamental & arbusto \\
\hline Schlumbergera truncata (Haw.) & flor de maio & $\mathrm{E}$ & ornamental & erva \\
\hline \multicolumn{5}{|l|}{ CARICACEAE } \\
\hline Carica papaya $\mathrm{L}$ & mamoeiro & $\mathrm{N}$ & alimenticia & árvore \\
\hline
\end{tabular}




\begin{tabular}{|c|c|c|c|c|}
\hline $\begin{array}{l}\text { ERICACEAE } \\
\text { Rhododendron indicum (L.) Sweet, }\end{array}$ & azaleia & $\mathrm{E}$ & ornamental & arbusto \\
\hline \multicolumn{5}{|l|}{ EUPHORBIACEAE } \\
\hline Euphorbia milii Des Moulins & coroa de cristo & $\mathrm{E}$ & ornamental & arbusto \\
\hline Codiaeum variegatum Baill. & cróton & $\mathrm{E}$ & ornamental & arbusto \\
\hline Phyllanthus niruri L. & quebra pedra & $\mathrm{N}$ & medicinal & erva \\
\hline \multicolumn{5}{|l|}{ FABACEAE } \\
\hline Albizia lebbeck (L.) Bentham & albizia & $\mathrm{E}$ & ornamental & árvore \\
\hline \multicolumn{5}{|l|}{ LAMIACEAE } \\
\hline Plectranthus barbatus Benth. & boldo & $\mathrm{E}$ & medicinal & erva \\
\hline Plectranthus forskohlii Willd. & boldinho & E & medicinal & erva \\
\hline Plectranthus amboinicus (Lour.) Spreng. & malvarisco & $\mathrm{N}$ & medicinal & erva \\
\hline \multicolumn{5}{|l|}{ LILIACEAE } \\
\hline Dracena marginata & dracena & E & ornamental & erva \\
\hline Sansevieria guineensis Wild. & espada de são jorge & E & ornamental & erva \\
\hline Aloe vera $\mathrm{L}$. & babosa & $\mathrm{N}$ & medicinal & erva \\
\hline Allium sativum $\mathrm{L}$. & alho & $\mathrm{E}$ & medicinal & erva \\
\hline Allium schoenoprasum $\mathrm{L}$. & cebolinha & $\mathrm{E}$ & medicinal & erva \\
\hline \multicolumn{5}{|l|}{ LOGANIACEAE } \\
\hline Nerium oleander L. & espirradeira & E & ornamental & arbusto \\
\hline \multicolumn{5}{|l|}{ MALVACEAE } \\
\hline Hibiscus sabdariffa $\mathrm{L}$. & hibisco & E & medicinal & erva \\
\hline \multicolumn{5}{|l|}{ MUSACEAE } \\
\hline Ravenala madagascariensis Sonn. & árvore do viajante & E & ornamental & árvore \\
\hline Musa paradisiaca $\mathrm{L}$. & banana & $\mathrm{E}$ & alimentícia & arbusto \\
\hline \multicolumn{5}{|l|}{ MYRTACEAE } \\
\hline Psidium guajava $\mathrm{L}$. & goiaba & $\mathrm{N}$ & alimentícia & árvore \\
\hline Myrciaria cauliflora Berg. & jaboticaba & $\mathrm{N}$ & medicinal & árvore \\
\hline Myrtus communis L. & murta & $\mathrm{N}$ & ornamental & árvore \\
\hline Eugenia uniflora $\mathrm{L}$. & pitanga & $\mathrm{N}$ & medicinal & árvore \\
\hline \multicolumn{5}{|l|}{ NYCTAGINACEAE } \\
\hline Bougainvillea spectabilis Willd. & bougavilia & $\mathrm{N}$ & ornamental & arbusto \\
\hline \multicolumn{5}{|l|}{ OLEACEAE } \\
\hline Jasminum officinale $\mathrm{L}$. & jasmim & $\mathrm{E}$ & ornamental & arvore \\
\hline \multicolumn{5}{|l|}{ POACEAE } \\
\hline Cymbopogon citratus (DC) Stapf. & capim limão & $\mathrm{E}$ & medicinal & erva \\
\hline Zoysia japonica Steud. & grama esmeralda & $\mathrm{E}$ & ornamental & erva \\
\hline \multicolumn{5}{|l|}{ POLYGONACEAE } \\
\hline Pilea nuттиlariifolia (Sw.) Wedd. & dinheiro em penca & E & ornamental & erva \\
\hline Pleopeltis pleopeltifolia (Raddi) & samambaia & E & ornamental & erva \\
\hline \multicolumn{5}{|l|}{ PORTULACACEAE } \\
\hline Portulaca oleracea $\mathrm{L}$. & onze horas & $\mathrm{N}$ & ornamental & erva \\
\hline \multicolumn{5}{|l|}{ PUNICACEAE } \\
\hline Punica granatum $\mathrm{L}$. & romã & $\mathrm{N}$ & medicinal & arvore \\
\hline \multicolumn{5}{|l|}{ RUBIACEAE } \\
\hline Citrus aurantium $\mathrm{L}$. & laranja & E & alimentícia & árvore \\
\hline Citrus limon $\mathrm{L}$. & limão & $\mathrm{E}$ & alimentícia & árvore \\
\hline Ixora coccínea $\mathrm{L}$. & ixora & $\mathrm{E}$ & ornamental & arbusto \\
\hline Mussaenda erythrophylla Schumach. \& Thonn. & mussaenda & $\mathrm{E}$ & ornamental & arbusto \\
\hline \multicolumn{5}{|l|}{ RUTACEAE } \\
\hline Ruta graveolens $\mathrm{L}$. & arruda & E & medicinal & erva \\
\hline \multicolumn{5}{|l|}{ VERBENACEAE } \\
\hline Lantana camara $\mathrm{L}$. & lantana & $\mathrm{E}$ & ornamental & erva \\
\hline Duranta repens L. & pingo de ouro & $\mathrm{E}$ & ornamental & erva \\
\hline \multicolumn{5}{|l|}{ ZINGIBERACEAE } \\
\hline Alpinia purpurata (Vieill.) K. Schum & alpinia & $\mathrm{E}$ & ornamental & erva \\
\hline
\end{tabular}

A família com maior número de espécies foi Arecaceae, assemelhando-se ao estudo de Pereira e Figueira Neto (2015) em Cáceres, Mato Grosso. Entretanto, outros estudos verificaram maior número de espécies na família Lamiaceae e Asteraceae, como nos estados do Espírito Santo (ALBERTASSE et al., 2010), Goiás (SILVA; PROENÇA, 2008), Maranhão (MONTELES; PINHEIRO, 2007), Minas Gerais (CALÁBRIA et al., 2008), Paraná (NEGRELLE et al., 2007), Rio Grande do Sul (VENDRUSCOLO; MENTZ, 2006), Rondônia (SANTOS et al., 2008), Santa Catarina (GIRALDI; HANAZAKI, 2010), São Paulo (MOREIRA; GUARIM NETO, 2015; PILLA et al., 2006). Ressalta-se que a maioria das espécies destas famílias apresenta compostos secundários com ação antimicrobiana e anti-inflamatória, dentre outras funções (ALMASSY et al., 2005), com efeitos comprovados por estudos científicos (LORENZI; MATOS, 2008).

O uso ornamental representou 62,5\%, o medicinal $21,5 \%$ e o alimentício apenas $16 \%$. Pedroga (2003) em um estudo na comunidade Clarinópolis, município de CáceresMT, encontrou uma tendência de distribuição de categorias semelhante em 215 espécies dos quintais estudados, sendo que $45,11 \%$ foram para uso ornamental, $21,39 \%$ para o uso medicinal e $19,53 \%$ alimentícia.

Estes dados diferem dos relatados em estudos conduzidos em Mato Grosso (AMARAL; GUARIM NETO, 2008; CARNIELLO et al. 2010; PASA, 2011; MOREIRA; GUARIM NETO, 2015) que encontraram como uso mais frequente as espécies alimentícias e medicinais. Possivelmente isto se deve a origem dos habitantes do bairro 
Jardim Florianópolis que não têm histórico de vida rural e, portanto, pouca tradição de convivência com as plantas, concentrando seu interesse em espécies ornamentais.

A predominância das plantas ornamentais em quintais urbanos foi constatada por outros estudos, como é o caso dos quintais em Rio Claro, nos quais Eichemberg et al. (2009) encontraram uma proporção maior de espécies ornamentais (63\%), seguidas das alimentícias (24\%) e por último as medicinais (23\%). Amorozo (2002) apontou para essa tendência nas cidades industrializadas do interior de São Paulo, com maiores taxas de plantas ornamentais. Nesse mesmo aspecto, foi observado que Boituva, Itapetininga, Porto Feliz e Mairiporã apresentaram as maiores taxas de plantas ornamentais $(47 \%, 35 \%, 30 \%$ e $29 \%$, respectivamente), sendo que estas também apresentaram as maiores taxas para alimentícias $(53 \%, 50 \%, 49 \%$ e $66 \%$, respectivamente).

Em outros estudos realizados em quintais a preparação na forma de chá (decocção) com as folhas foi a mais citada (ALBERTASSE et al., 2010; BATTISTI et al., 2013), como no presente trabalho.

As espécies usadas na alimentação são também as mais frequentes nos estudos sobre quintais no Brasil: Anacardium ocidentalis, Annona squamosa, Carica papaya, Citrus aurantium, Citrus limon, Cocos nucifera, Mangifera indica, Musa sapientum e Psidium guajava.

Em Rosário Oeste-MT Amaral \& Guarim Neto (2008) identificaram 94 espécies vegetais cultivadas com a finalidade de complementação da dieta alimentar representadas por espécies frutíferas, como Anacardium ocidentalle, Carica papaya e Musa paradisiaca.

As espécies de uso medicinal embora sejam pouco frequentes representam um papel importante nos cuidados com a saúde. Verifica-se que sintomas comuns nas populações como gripes, resfriados e inflamações são tratados com chás (Tabela 2).

Tabela 2. Espécies de uso medicinal levantadas nos quintais do Bairro Jardim Florianópolis em Cuiabá, Mato Grosso

\begin{tabular}{ll}
\hline Espécies & Usos \\
\hline Allium sativum & Chá junto com limão para gripe \\
Allium schoenoprasum & Chá para gripe \\
Aloe vera & $\begin{array}{l}\text { Gel para queimaduras e para lavar os } \\
\text { cabelos }\end{array}$ \\
Cymbopogon citratus & Chá para dormir bem, calmante \\
Eugenia uniflora & Chá das folhas para diarreia \\
Hibiscus sabdariffa & Chá para emagrecer e baixar a pressão \\
Myrciaria cauliflora & Chá das folhas para pressão alta \\
Plectranthus barbatus & Chá para o fígado e indigestão \\
Plectranthus forskohlii & Chá para o fígado e indigestão \\
Plectranthus amboinicus & Lambedor para gripe e dor de garganta \\
Psidium guajava & Chá das folhas jovens para diarreia \\
Punica granatum & Chá da casca do fruto para dor de garganta \\
Ruta graveolens & Chá das folhas para lavar os olhos quando \\
\hline
\end{tabular}

As espécies pertencentes à família Lamiaceae como Plectranthus amboinicus, Plectranthus barbatus $e$ Plectranthus forskohlii são também citadas em vários outros estudos com quintais em Mato Grosso (AMARAL; GUARIM NETO, 2008; GUARIM NETO; CARNIELLO, 2008; CARNIELLO et al., 2010; PASA, 2011; MOREIRA; GUARIM NETO, 2015), sendo esta família uma das principais fornecedoras de plantas usadas como medicinal em todo o mundo (ABDEL-MOGIB et al., 2002).

\section{CONCLUSÕES}

Nos quintais agroflorestais do Bairro Jardim Florianópolis as plantas são classificadas pelos moradores em ornamentais, alimentícias e medicinais. As espécies ornamentais de hábito herbáceo são as mais importantes com predominância da família Arecaceae.

\section{REFERÊNCIAS}

ABDEL-MOGIB, M.; ALBAR, H. A.; BATTERJEE, S. M. Chemistry of the Genus Plectranthus. Molecules, v. 7, p. 271301, 2002.

ALBERTASSE, P. D.; THOMAZ, L. D.; ANDRADE, M. A. Plantas medicinais e seus usos na comunidade da Barra do Jucu, Vila Velha, ES. Revista Brasileira de Plantas Medicinais, v.12, n.3, p.250-260, 2010.

ALBUQUERQUE, U. P.; CUNHA, L. V. F. C.; LUCENA, R. F. P.; ALVES, R. R. N. (Eds.) Methods and Techniques in Ethnobiology and Ethnoecology, Springer Protocols Handbooks, 2014, 480p.

ALMASSY, J.A.A.; LOPES, R.C; ARMOND, C.; SILVA, F.; CASALI, V.W.D. Folhas de Chá: Plantas Medicinais na Terapêutica Humana. Viçosa: Ed. UFV, 2005, 233p.

AMARAL, C. N.; NETO, G. G. Os quintais como espaços de conservação e cultivo de alimentos: um estudo na cidade de Rosário Oeste (Mato Grosso, Brasil). Boletim do Museu Paraense Emílio Goeldi. Ciências Humanas, v.3, n.3, p.329$341,2008$.

AMOROZO, M. C. M. Uso e Diversidade de Plantas Medicinais em Santo Antônio do Leverger, MT, Brasil. Acta Botânica Brasílica, v.16, n.2, p.189-203, 2002.

AMOROZO, M. C. M.; VIERTLER, R. B. A abordagem qualitativa na coleta e análise de dados em etnobiologia e etnoecologia. In: ALBUQUERQUE, U.P.; LUCENA, R.F.P.; CUNHA, L.V.F.C. Métodos e técnicas na pesquisa etnobiológica e etnoecológica. Recife: NUPEEA, 2010, p.6782.

BAILEY, K. D. Methods of Social Research. 4. ed. New York: The Free Press, 1994, 588p.

BAJPAI, S.; SHARMA, A. K.; KANUNGO, V. K. Traditional home gardens: A preserve of medicinal plants. International Journal of Herbal Medicine, v.1, n.2, p. 152$161,2013$.

BATTISTI, C.; GARLET, T. M. B.; ESSI, L.; HORBACH, R. K.; ANDRADE, A.; BADKE, M. R. Plantas medicinais utilizadas no município de Palmeira das Missões, RS, Brasil. Revista Brasileira de Biociências, v.11, n.3, p.338-348, 2013.

CALÁBRIA, L.; CUBA, G. T.; HWANG, S. M.; MARRA, J. C. F.; MENDONÇA, M. F.; NASCIMENTO, R. C.; OLIVEIRA, M. R.; PORTO, J. P. M.; SANTOS, D. F.; 
SILVA, B. L.; SOARES, T. F.; XAVIER, E. M.; DAMASCENO, A. A.; MILANI, J. F.; REZENDE, C. H. A.; BARBOSA, A. A. A.; CANABRAVA, H. A. N. Levantamento etnobotânico e etnofarmacológico de plantas medicinais em Indianópolis, Minas Gerais, Brasil. Revista Brasileira de Plantas Medicinais, v.10, n.1, p.49-63, 2008.

CARMO, E. L. I. A Vivência e o Bairro: O Jardim Florianópolis em Cuiabá - Mato Grosso In: Anais VII Congresso Brasileiro de Geógrafos, 2014, ISNB 978-8598539-04-1 Disponível em: http://www.cbg2014.agb.org.br/resources/anais/1/140538815 9_ARQUIVO_EdsontxtcompletoCBG2014.pdf Acesso em: nov 2015.

CARNIELlO, M. A.; SILVA, R. D. S.; CRUZ, M. A. B. D.; GUARIM NETO, G. Quintais urbanos de Mirassol D’OesteMT, Brasil: uma abordagem etnobotânica. Acta Amazônica, v.40, n.3, p. 451-470, 2010.

EICHEMBERG, M. T.; AMOROZO, M. C. M.; MOURA, L. C. Species composition and plant use in old urban homegardens in Rio Claro, Southeast of Brazil. Acta Botânica Brasílica, v.23, n.4, p.1057-1075, 2009.

FLORENTINO, A.T.N.; ARAUJO, E.L.; ALBUQUERQUE, U.P. Contribuição de quintais agroflorestais na conservação de plantas da Caatinga, Município de Caruaru, PE, Brasil. Acta Botânica Brasílica, v.21, n.1, p.37-47, 2007.

GAO, J.; HE, T.; LI, Q. M. Traditional home-garden conserving genetic diversity: a case study of Acacia pennata in southwest China. Conservation Genetics, v.13, n.4, p.891898, 2012.

GIRALDI, M.; HANAZAKI, N. Uso e conhecimento tradicional de plantas medicinais no Sertão do Ribeirão, Florianópolis, SC, Brasil. Acta Botânica Brasílica, v.24, n.2, p.395-406, 2010.

GUARIM NETO, G.; CARNIELLO, M. A. Quintais matogrossenses: espaços de conservação e reprodução de saberes. Mato Grosso: UNEMAT, 2008. 201p.

KUMAR, B. M.; NAIR, P. K. R. Tropical home gardens: A time tested example of sustainable agroforestry. Netherlands: Springer Science, 2006. 380p.

LORENZI, H.; MATOS, F. J. A. M. Plantas Medicinais no Brasil: nativas e exóticas. 2.ed. Nova Odessa: Instituto Plantarum, 2008. 544p.

MONTELES, R.; PINHEIRO, C. U. B. Plantas medicinais em um quilombo maranhense: uma perspectiva etnobotânica. Revista de Biologia e Ciências da Terra, v.7, n.2, p.38-48, 2007.
MOREIRA, R. P. M.; GUARIM NETO, G. A flora medicinal dos quintais de Tangará da Serra, Mato Grosso, Brasil. Biodiversidade, v.14, n.1, p.63-83, 2015.

NEGRELLE, R. R. B.; TOMAZZONI, M. I; CECCON, M. F; VALENTE, T. P. Estudo etnobotânico junto à Unidade Saúde da Família Nossa Senhora dos Navegantes: subsídios para o estabelecimento de programa de fitoterápicos na Rede Básica de Saúde do município de Cascavel (Paraná). Revista Brasileira de Plantas Medicinais, v.9, n.3, p.6-22, 2007.

PASA, M. C. Saber local e medicina popular: a etnobotânica em Cuiabá, Mato Grosso, Brasil. Boletim do Museu Paraense Emílio Goeldi. Ciências Humanas, v.6, n.1, p.179-196, 2011.

PEDROGA, J. A. Estudo de Plantas nos Quintais: comunidade Clárinopolis Cáceres, Mato Grosso. 2003. 52f. Monografia (Graduação em Ciências Biológica). Universidade do Estado de Mato Grosso. Cáceres, 2003.

PEREIRA, P. V. M.; FIGUEIREDO NETO, L.F. Conservação de espécies florestais: um estudo em quintais agroflorestais no município de Cáceres - MT. Revista Eletrônica em Gestão, Educação e Tecnologia Ambiental, v.19, n.3, p.783-793, 2015.

PERNA, T. A.; LAMANO-FERREIRA, A. P. N. Revisão Bibliométrica Sobre o Cultivo de Plantas Medicinais em Quintais Urbanos em Diferentes Regiões do Brasil (20092012). UNOPAR Científica Ciências Biológicas e da Saúde, v.16, n.1, p.61-67, 2014.

PILlA, M. A. C.; AMOROZO, M. C. M.; FURLAN, A. Obtenção e uso das plantas medicinais no distrito de Martim Francisco, Município de Mogi-Mirim, SP, Brasil. Acta Botânica Brasílica, v.20, n.4, p.789-802, 2006.

SANTOS, J. F. L.; AMOROZO, M. C. M.; MING, L. C. Uso popular de plantas medicinais na comunidade rural da Vargem Grande, Município de Natividade da Serra, SP. Revista Brasileira de Plantas Medicinais, v.10, n.3, p.67$81,2008$.

SILVA-ALMEIDA, M. F.; AMOROZO, M. C. M. Medicina popular no Distrito de Ferraz, Município de Rio Claro, SP. Brazilian Journal of Ecology, v.2, n.1, p.36-46, 1998.

SILVA, C. S. P.; PROENÇA, C. E. B. Uso e disponibilidade de recursos medicinais no município de Ouro Verde de Goiás, GO, Brasil. Acta Botânica Brasílica, v.22, n.2, p.481-92, 2008.

VENDRUSCOLO, G. S.; MENTZ, L. A. Levantamento etnobotânico das plantas utilizadas como medicinais por moradores do bairro Ponta Grossa, Porto Alegre, Rio Grande do Sul, Brasil. IHERINGIA, Série Botânica, v.61, n.1-2, p.83103, 2006. 